\title{
Testosterontherapie effektiv bei hypogonadalen Männern
}

- Männer, die unter Hypoganadismus leiden, haben mit ernst zu nehmenden Symptomen zu kämpfen. Anfangs äußert sich der Testosteronmangel meist mit einem Verlust der Antriebskraft und einer verringerten Libido. Je niedriger die Testosteronkonzentration, desto mehr Beschwerden beklagen die Patienten im, ,aging male syptom's score“ (AMS-Fragebogen).

Sehr häufig ist Übergewicht mit einem Testosteronmangel assoziiert: Über die Hälfte aller übergewichtigen Männer sind betroffen.

Als Therapie des Hypogonadismus ist eine Testosteronsubstitution möglich. Diese Behandlung kann nicht nur die sexuelle
Funktion verbessern, sondern außerdem die kardiovaskulären Risikofaktoren günstig beeinflussen, die Knochendichte erhöhen und die Stimmung verbessern. Bei älteren hypogonadalen Männern führt die Substitution mit Testosterongel (z.B. mit Testogel ${ }^{\circledR}$ ) zur Besserung depressiver Symptome, selbst wenn diese bereits mit Antidepressiva behandelt werden, wie eine Studie von Pope et al. gezeigt hat.

Einen anderen Aspekt des Hypogonadismus beleuchtete eine Studie mit gebrechlichen Männern im Alter von über 65 Jahren: Durch die Testosteronsubstitution erhöhte sich ihre 6-Minuten-Gehstrecke um 30 bis 40 Meter. „Dies ist eine klinisch relevante Verbesserung, die darauf beruht, dass sich durch die Testosteronsubstitution die Körperzusammensetzung im Sinne einer Zunahme der Muskelmasse verändert hat“, erklärt Prof. Dr. Michael Zitzmann, Münster.

Viele Ärzte schrecken aus Angst vor einem Prostatakarzinom vor einer Testosterontherapie zurück. Eine Metaanalyse von 18 prospektiven Studien hat jedoch gezeigt, dass es keinen Zusammenhang zwischen Serumtestosteronkonzentration und Prostatakarzinom gibt.

Nach Informationen von

Bayer Vital, Leverkusen

\section{Reine Hyaluronsäure für gesunde Harnwege}

_ Eine Behandlung mit reiner Hyaluronsäure (Cystistat ${ }^{\circledR}$ ) ist bei Interstitieller Zystitis (IC) sowie bei anderen Zystitiden wie der chronischen Zystitis, der radiogenen Zystitis und der Zystitis, die mit einer abak- teriellen Prostatitis bei Männern verbunden ist, erfolgversprechend. Das Unternehmen Uromed hat diese in der Anwendung bereits seit langem bewährte Hyaluronsäurefraktion mit hoher Reinheit kürzlich exklu-

\section{Benigne Prostatahyperlasie: EU-Zulassung für Kombinationspräparat}

_ GlaxoSmithKline hat Ende März die EUZulassung für Duodart ${ }^{\circledR}$ erhalten. Dieses Kombinationspräparat aus den Wirkstoffen Dutasterid (0,5 mg) und Tamsulosin (0,4 mg) ist für die Behandlung der mittleren bis schweren Symptome einer benignen Prostatahyperplasie (BPH) geeignet. Außerdem ist die Kombination für die Reduzierung des Risikos für akuten Harnverhalt (AUR) sowie für BPH-bedingte Operationen zugelassen. Eddie Gray, Präsident von Pharma Europe, GlaxoSmithKline betont: „GlaxoSmithKline hat den Nutzen der beiden Substanzen und ihre synergetische Wirkung erkannt und diese neue Behandlungsalternative entwickelt, um Ärzten und ihren Patienten eine komfortable Therapie mit einer täglichen Einmal-Dosis zu geben, die sowohl effektiv die Symptome dieser häufigen Erkrankung als auch das Risiko von möglichen Komplikationen bis hin zur Operation reduziert.“
Der Zulassungsantrag zu Duodart ${ }^{\circledR}$ basierte auf den Daten der CombAT-Studie, die gezeigt hat, dass das Kombinationspräparat bei BPH folgende Vorteile bringt:

- signifikante und anhaltende Verbesserung der Symptome im Vergleich zum Alphablocker Tamsulosin,

- Symptomverbesserung, die genauso schnell wie unter Therapie mit Tamsulosin eintritt, dabei aber über mindestens vier Jahre beibehalten wird, und

- eine Risikoreduktion von mit einer $\mathrm{BPH}$ verbundenen Komplikationen. Generell wurde die Kombinationstherapie gut vertragen und die meisten Nebenwirkungen entsprachen den bekannten Sicherheitsprofilen der Einzelsubstanzen.

Nach Informationen von GlaxoSmithKline, München siv in sein Produktprogramm aufgenommen.

Die Wirksamkeit von Cytistat ${ }^{\circledR}$ wurde in circa 40 Studien mit insgesamt über 1.000 Patienten untersucht. Beispielsweise konnte in einer Studie bei der Behandlung von 121 IC-Patienten mit Hyaluronsäure bei 103 Patienten (85\%) eine Verbesserung der Symptome festgestellt werden. Auf einer Skala von o bis 10 hatten die Patienten ihre Beschwerden zuvor im Schnitt mit 8,5 bewertet - nach der Therapie lag dieser Wert bei 3,5. Bei $55 \%$ der Patienten war auch fünf Jahre nach Therapieende keine weitere Behandlung notwendig geworden [RiedI CR et al. Hyaluronan treatment of interstitial cystitis/painful bladder syndrome. Int Urogynecol J Pelvic Floor Dysfunct 2008; 19: 717-21].

Eine weitere Studie zeigte, dass sich die durchschnittliche Dauer bis zum erneuten Auftreten von Symptomen bei Patienten mit chronischer Zystitis nach der Behandlung mit Hyaluronsäure um das Fünffache verlängerte. Die Patienten waren im Schnitt 498 Tage symptomfrei - gegenüber 96 Tagen vor der Behandlung [Constantinides $C$ et al. Prevention of recurrent bacterial cystitis by intravesical administration of hyaluronic acid: a pilot study. BJU Int 2004; 93: 1262-6].

Nach Informationen von

Uromed, Oststeinbek 